\title{
Changing expression and subcellular distribution of karyopherins during murine oogenesis
}

\author{
Bettina P Mihalas ${ }^{1,3}$, Patrick S Western ${ }^{2,4}$, Kate L Loveland ${ }^{2,4}$, Eileen A McLaughlin ${ }^{3}$ \\ and Janet $\mathrm{E}$ Holt $^{1}$ \\ ${ }^{1}$ School of Biomedical Sciences and Pharmacy, University of Newcastle, Callaghan, New South Wales 2308, \\ Australia, ${ }^{2}$ Hudson Institute of Medical Research, Clayton, Victoria 3168, Australia, ${ }^{3}$ School of Environmental and Life \\ Sciences, University of Newcastle, Callaghan, New South Wales 2308, Australia and ${ }^{4}$ School of Clinical Sciences and \\ Department of Anatomy and Developmental Biology, Monash University, Clayton, Victoria 3800, Australia
}

Correspondence should be addressed to J E Holt; Email: janet.holt@newcastle.edu.au

\begin{abstract}
Mammalian oocyte growth and development is driven by a strict program of gene expression that relies on the timely presence of transcriptional regulators via nuclear pores. By targeting specific cargos for nucleo-cytoplasmic transport, karyopherin (KPN) proteins are key to the relocation of essential transcription factors and chromatin-remodelling factors into and out of the nucleus. Using multiple complementary techniques, here we establish that KPNA genes and proteins are dynamically expressed and relocalised throughout mouse oogenesis and folliculogenesis. Of the KPNAs examined (Kpna1, Kpna2, Kpna3, Kpna4, Kpna6, Kpna7, Kpnb1, Ipo5 and Xpo1), all were expressed in the embryonic ovary with up-regulation of protein levels concomitant with meiotic entry for KPNA2, accompanied by the redistribution of the cellular localisation of KPNA2 and XPO1. In contrast, postnatal folliculogenesis revealed significant up-regulation of Kpna1, Kpna2, Kpna4, Kpna6 and Ipo5 and down-regulation of Kpnb1, Kpna7 and Xpo1 at the primordial to primary follicle transition. KPNAs exhibited different localisation patterns in both oocytes and granulosa cells during folliculogenesis, with three KPNAs - KPNA1, KPNA2 and IPO5 - displaying marked enrichment in the nucleus by antral follicle stage. Remarkably, varied subcellular expression profiles were also identified in isolated pre-ovulatory oocytes with KPNAs KPNA2, KPNB1 and IPO5 detected in the cytoplasm and the nuclear rim and XPO1 in cytoplasmic aggregates. Intriguingly, meiotic spindle staining was also observed for KPNB1 and XPO1 in meiosis II eggs, implying roles for KPNAs outside of nucleo-cytoplasmic transport. Thus, we propose that KPNAs, by targeting specific cargoes, are likely to be key regulators of oocyte development.
\end{abstract}

Reproduction (2015) 150 485-496

\section{Introduction}

Mammalian female germ cells follow a unique path of growth and development that features several periods of cell cycle pausing and culminates in the formation of a mature meiosis II (MII)-arrested egg capable of being fertilised. Female meiosis is initiated during fetal life, around embryonic day 13.5 (E13.5) in the mouse, with oogonia arresting in meiotic prophase I prior to birth. In the postnatal ovary, somatic cells invade nests of oogonia to enclose individual oocytes in primordial follicles. Oocytes remain quiescent in this structure until recruited to enter the growing follicle pool, where development from a preantral to a mature pre-ovulatory follicle occurs. Despite being arrested at prophase I, the oocyte retains the capacity for significant growth to become a transcriptionally quiescent pre-ovulatory germinal vesicle (GV) oocyte. Upon ovulation, MI resumes, with a concordant breakdown of the nuclear envelope and condensation of chromatin. Homologous chromosomes are then segregated during an asymmetric cytokinesis in which the first polar body, containing half the chromosomal complement, is extruded and the egg again arrests at metaphase II prior to fertilisation (Hirshfield 1991, McLaughlin \& Mclver 2009, Holt et al. 2013).

A strict program of regulated gene expression and nuclear-cytoplasmic transport of cell cycle proteins governs oocyte development. Because an intact nuclear envelope is present until meiotic resumption, the majority of macromolecules $>45 \mathrm{kDa}$ must traffic through nuclear pore complexes to and from the nucleus by an active, GTP-dependent mechanism. Karyopherins (KPNs/Thermofisher Scientific, Waltham, MA, USA) represent the major class of nuclear transport factors that interact with the nuclear pores to perform nucleocytoplasmic shuttling of protein and RNA cargoes (Macara 2001, Stewart 2007). More than 20 KPNA genes have been identified in the human genome with equivalent homologs conserved in mouse. Each encoded protein binds a distinct subset of cargo proteins through the recognition of nuclear localisation (NLS) or nuclear 
export signal (NES) sequences. KPNAas, also known as importin $\alpha$, function predominantly as importers, whereas KPNA $\beta$ s, also known as importin $\beta$ s and exportins, have roles in both nuclear import and export (Major et al. 2011, Marfori et al. 2011). Within the KPNA $\alpha$ family, Kpna 1 and Kpna6 share a high level of identity (81\%), as do Kpna3 and Kpna4 (85\%) (Holt et al. 2007).

In addition to nucleo-cytoplasmic trafficking roles, KPNAs have also been shown to function in other cellular processes of metazoans including mitotic spindle organisation (Gruss et al. 2001, Gorjanacz et al. 2002, Schatz et al. 2003, Ciciarello et al. 2004, Ems-McClung et al. 2004) and nuclear envelope and nuclear pore complex formation (Geles et al. 2002, Zhang et al. 2002, Harel et al. 2003, Tirian et al. 2003). More recently, roles have been described for Kpna2 and Kpna4 in somatic and germ cell responses to oxidative stress respectively, which appears to be through a direct influence on gene expression (Yasuda et al. 2012, Young et al. 2013).

Nucleo-cytoplasmic transport has been implicated as a driver of germ cell differentiation in the mammalian testes, with changes in KPNA expression and subcellular localisation correlated with developmental milestones during rodent and human spermatogenesis (Hogarth et al. 2007, Itman et al. 2009, Major et al. 2011, Miyamoto et al. 2012, Whiley et al. 2012). Emerging evidence, including phenotypes of KPNA knockout mouse models, suggests there may be a corresponding role for this protein family in the female germline. KPNA7 is expressed in oocytes and appears to be important for pre-implantation development with parthogenetically activated eggs from conditional mutants failing to develop to the blastocyst stage (Hu et al. 2010). The absence of KPNA1, also known as importin $\alpha 5$, leads to reduced numbers of growing follicles and hypoplasia of the reproductive tract in mice (Moriyama et al. 2011). KPNA6 in contrast acts as a maternal effect gene required for zygotic genome activation (Rother et al. 2011). However, there is limited expression data about other KPNA family members in the ovary and specifically in the oocyte. In the current study, we sought to fill this knowledge gap by undertaking in silico analysis of KPNA transcripts and then examining protein expression in mouse oocytes from embryonic life through to the mature ovulated egg. To provide a meaningful comparison with another developmental system, we focused primarily on a cohort of KPNAs that are known to be expressed during male germ cell differentiation and in the developing and adult testis (Hogarth et al. 2006, 2007, Loveland et al. 2006, Major et al. 2011, Whiley et al. 2012).

\section{Materials and methods}

All reagents were obtained from Sigma-Aldrich unless otherwise specified.

\section{Animals}

Use of animals for this study was approved by the University of Newcastle Animal Care and Ethics Committee, Australia. The C57BL/6J strain was used and animals were held under a $12 \mathrm{~h}$ light:12 $\mathrm{h}$ darkness cycle and provided with a regular supply of food and water and allowed to feed ad libitum.

\section{Tissue collection and histology}

Reproductive organs were fixed in Bouin's solution, paraffinembedded and sectioned at 5-8 $\mu \mathrm{m}$ prior to immunohistological processing.

\section{Embryonic germ cell isolation}

Germ cell isolation from embryos at E12.5 and E14.5 was performed as per van den Bergen et al. (2009). Briefly, embryos were derived from OG2 (Oct4 (Pou5f1)-eGFP) transgenic male (C57Bl6) $\times$ CD1 female matings; $6-18$ ovaries (age dependent) were pooled and dissociated with trypsin. Enhanced green fluorescent protein (EGFP)-positive and -negative cells were isolated using fluorescence-activated cell sorting. Tissues were collected in biological triplicate.

\section{Oocyte collection}

An i.p. injection of $10 \mathrm{IU}$ of pregnant mares serum gonadotrophin was administered $44-52 \mathrm{~h}$ before collection of GV oocytes from 4- to 6-week-old mice, or a second injection of $5 \mathrm{IU}$ hCG was given prior to MII collection. Oocytes were released into M2 media with 4\% BSA, and cumulus cells were mechanically removed by repeated pipetting (GV oocytes) or enzymatically removed by a brief incubation in M2 with hyaluronidase $(300 \mu \mathrm{g} / \mathrm{ml})$. Oocytes and eggs were fixed in $4 \%$ paraformaldehyde/PBS/0.5\% Triton-X for $20 \mathrm{~min}$ prior to immunostaining.

\section{Oocyte and germ cell RT-PCR}

Five denuded GV oocytes were used per reaction. Oocytes were briefly incubated in acidified Tyrode's solution to remove the zona pellucida washed in PBS/polyvinylpyrrolidone and transferred into $5 \mu \mathrm{l}$ mRNA lysis buffer $(5 \mathrm{mM}$ dithiothreitol and $20 \mathrm{U} / \mathrm{ml}$ RNase inhibitor; Promega) and 1\% NP-40 (w/v). Samples were frozen and thawed three times before incubation with $1 \mu \mathrm{l}$ DNase (Promega) at $37^{\circ} \mathrm{C}$ for $15 \mathrm{~min}$. DNase was inactivated by incubating the reactions at $75^{\circ} \mathrm{C}$ for $5 \mathrm{~min}$. First strand cDNA synthesis was then completed using moloney murine leukaemia virus reverse transcriptase (Promega) with oligo $d(T)$ primers. RNA was extracted from germ cells as described by van den Bergen et al. (2009). Briefly, RNA was extracted using an RNeasy Micro Purification Kit (Qiagen) as per the manufacturer's instructions. High quality RNA underwent a single-round amplification reaction using the MessageAmp II antisense RNA (aRNA) Amplification Kit (Ambion/Thermofisher Scientific, Waltham, MA, USA) as per the manufacturer's instructions. Using random hexamers and Superscript III (Invitrogen), $100 \mathrm{ng}$ of aRNA was reverse 
transcribed, as per the manufacturer's instructions. Four microliters of oocyte cDNA template and $1 \mu$ of germ cell cDNA template was used per PCR, which was performed with GoTaq DNA Polymerase (Promega) using the following thermocycling conditions: $95^{\circ} \mathrm{C}$ for 2 min, 35 cycles of $95{ }^{\circ} \mathrm{C}$ for $30 \mathrm{~s}, x^{\circ} \mathrm{C}$ for $30 \mathrm{~s}$ and $72{ }^{\circ} \mathrm{C}$ for $5 \mathrm{~min}$, with a final extension at $72{ }^{\circ} \mathrm{C}$ for $5 \mathrm{~min}$, where ' $x$ ' is annealing time specific to each primer pair, as listed in Supplementary Table S2, see section on supplementary data given at the end of this article. The majority of primers sequences were based on those of Hogarth et al. (2006) and Loveland et al. (2006).

\section{TaqMan real-time PCR assay}

Real-time PCRs were performed on the LightCycler 96 RealTime PCR System (Roche) using TaqMan Gene Expression Master Mix (Life Technologies) as per the manufacture's instructions; $1 \mu \mathrm{l}$ of germ cell cDNA template was used per PCR, and peptidylprolyl isomerase A (Ppia) was used as an internal control. Real-time PCR was performed in biological and technical triplicate. The following thermocycling conditions were used: $50{ }^{\circ} \mathrm{C}$ for $2 \mathrm{~min}, 95^{\circ} \mathrm{C}$ for $10 \mathrm{~min}$ and 40 cycles of $95{ }^{\circ} \mathrm{C}$ for $15 \mathrm{~s}$ and $60{ }^{\circ} \mathrm{C}$ for $1 \mathrm{~min}$. Data analysis was generated by LightCycler 96 Real-Time PCR System Software using the $2^{-\Delta C T}$ method (Livak \& Schmittgen 2001).

\section{Gene Expression Omnibus data set analysis}

Microarray datasets used to a examine transcript expression were obtained from the National Center for Biotechnology Information (NCBI) websites (http://www.ncbi.nlm.nih.gov/). The following datasets were used: GSE5668 (Su et al. 2007), GSE27715 (Jameson et al. 2012) and GSE3351 (Pan et al. 2005). In cases in which a gene was represented by more than one oligonucleotide probe set, the expression profiles for each probe set was compared and a single set chosen to represent the gene. Detection Call=ABSENT in the data was considered uninformative and excluded from the data set. For each time point, the mean of three samples was determined from the probeset of each gene that showed the highest detection levels and/or least variation between replicates. The full list of Gene Expression Omnibus (GEO) datasets and the oligonucleotide probe set IDs used are shown in Supplementary Table S1, see section on supplementary data given at the end of this article.

\section{Immunoblotting}

Protein was extracted using RIPA lysis buffer with ProteCEASE Protease Inhibitor (G-Biosciences, St Louis, MO, USA) and quantitated using the Pierce BCA Protein Assay Kit (Thermo Scientific, Waltham, MA, USA). For oocytes/eggs, 50-100 oocytes/lane were used. Proteins were separated on a NuPage or Tris-Glycine 10\% gel (Life Technologies) blotted using an XCell Blot Module (Life Technologies). Primary antibodies used are listed in Supplementary Table S3, see section on supplementary data given at the end of this article. Anti-mouse/rabbit IgG HRP secondary antibodies were used (Dako, Glostrup, Denmark) and detection was performed using ECL reagents (GE Healthcare, Little Chalfont, Buckinghamshire, UK).
Densitometric analysis was performed using ImageJ (National Institutes of Health, Bethesda, MD, USA), normalised to GAPDH and reported as arbitrary units.

\section{Immunohistology and immunocytochemistry}

Primary antibodies used are listed in Supplementary Table S3. Secondary antibodies conjugated to Alexa Fluors 594 and 633 (Life Technologies) were used. Negative controls with only secondary antibodies were also examined. Counterstaining was performed with $0.2 \mu \mathrm{g} / \mathrm{ml} \mathrm{4}$ ',6-diamidino-2-phenylindole or Hoechst $33258(20 \mu \mathrm{g} / \mathrm{ml})$. Images were acquired using an Olympus FV1000 confocal microscope (Olympus, Japan) or Axio Imager A1 epifluorescent microscope (Carl Zeiss, North Ryde, NSW, Australia) with Olympus DP70 camera. Data analysis was performed using ImageJ (National Institutes of Health).

\section{Statistical analysis}

All statistical analysis was performed using GraphPad Prism Software (San Diego, CA, USA). Two-way unpaired student's $t$-tests, one-way ANOVA's with Dunn's multiple comparison tests were used.

\section{Results}

\section{Expression of KPNAs during embryonic female germ cell development}

Embryonic expression of key KPNAs (Kpna1, Kpna2, Kpna3, Kpna4, Kpna6, Kpna7, Kpnb1, Ipo5 and Xpo1) was established using RT-PCR on isolated female mouse germ cell mRNA (Fig. 1). Here we selected the developmental stages of E12.5, when germ cells are premeiotic, and E14.5, when the majority of cells are in early meiosis. All KPNAs were expressed at both time points, although notably, signal for Kpna4 and Kpna7 was low relative to the adult mouse testis positive control sample (Fig. 1). To gain a quantitative understanding of selected KPNA transcripts, data in the GEO (NCBI website) was then used to generate transcript profiles of KPNA family members during female germ cell development, similar to profiles established in the male germ line (Major et al. 2011). For embryonic germ cells, GEO series GSE27715 (Jameson et al. 2012) was analysed. This contains data from isolated cell populations encompassing the periods when primordial germ cells proliferate (E11.5), undergo sex determination in females (E12.5) and begin to enter meiosis in the female (E13.5) (Probeset IDs listed in Supplementary Table S1). Supplementary Figure 1, see section on supplementary data given at the end of this article illustrates the expression profiles for the KPNAas (Kpna1, Kpna2, Kpna3, Kpna4, Kpna6 and Kpna7) and KPNAßs (Kpnb1, Ipo5 and Xpo1). During this phase of embryonic ovary development, all selected KPNA transcripts were detected, with levels of most remaining relatively consistent across the time period. 
Kpna1

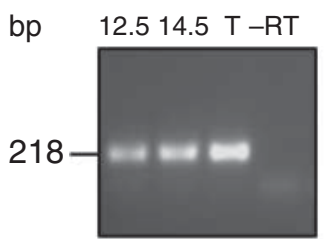

Kpna4

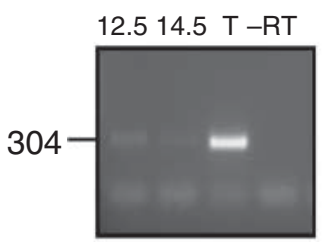

Kpnb1

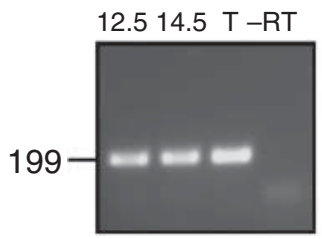

Kpna2

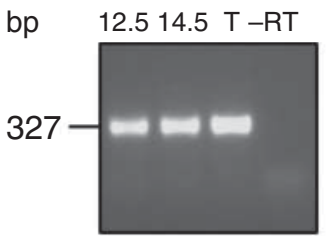

Kpna6

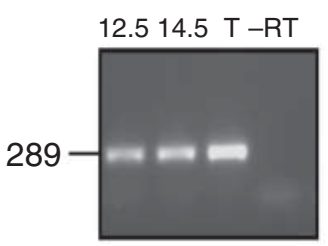

Ipo5

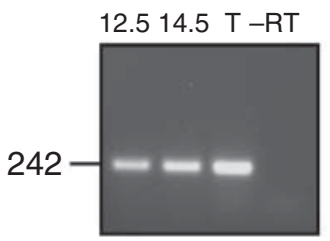

Kpna3

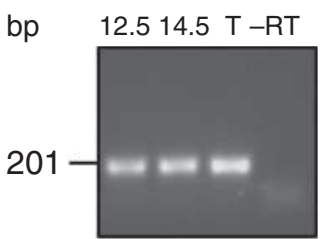

Kpna7

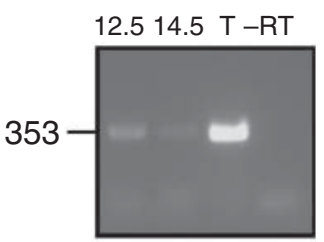

Xpo1

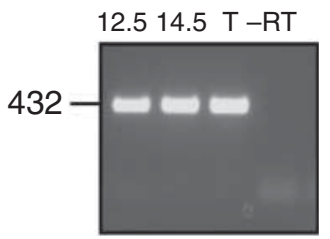

Figure 1 Expression of karyopherin transcripts in female mouse germ cells at E12.5 and E14.5. Representative RT-PCRs presented for Kpna1, Kpna2, Kpna3, Kpna4, Kpna6, Kpna7, Kpnb1, Ipo5 and Xpo1. RT-PCRs were performed in biological triplicate. $\mathrm{T}$, testis (positive control cDNA); RT, minus RT negative control.
We next sought to confirm whether these transcripts were translated during embryonic ovary development. Because insufficient protein samples precluded western blot analysis on isolated germ cells, we performed western blotting for KPNA1/6, KPNA2, KPNA3, KPNB1, IPO5 and XPO1 on whole embryonic gonads at E12.5 and E14.5. Although commercially available KPNA1/6 antibody is predicted to be specific to KPNA1, we noted $73 \%$ similarity $100 \%$ identity match between KPNA1 and KPNA6, for the 13 amino acid peptide antigen sequence, and therefore we chose not to distinguish between these two closely related KPNAs using this antibody. A suitable commercial antibody was unavailable for KPNA7. All proteins were detected at similar levels at both time points with the exception of KPNA2 (Fig. 2A), which displayed a twofold increase from E12.5 to E14.5 (Fig. 2D). This surprising increase in KPNA2 protein prompted us to examine mRNA transcript levels using TaqMan real-time analysis (Fig. 2E). Interestingly, we noted a small but significant decrease in Kpna2 transcript, suggesting that translational regulation may impact KPNA2 protein expression in the embryonic ovary.

\section{Distinct KPNA subcellular expression patterns in the female embryonic gonad}

As the focus of our study was the germ cell, we wanted to confirm whether KPNA protein was indeed expressed in the oogonia. We next used immunolocalisation to determine the subcellular localisation of KPNA1/6,
KPNA2, KPNA3, KPNB1, IPO5 and XPO1 in ovaries at E12.5 and E14.5 (Fig. 2B). KPNA1/6 was present predominantly in the cytoplasm of E12.5 and E14.5 germ cells and somatic cells, with oogonia identified by their large rounded nuclei (Fig. 2Bi and ii). In contrast, KPNA2 appeared restricted to the germ cells at E12.5 at which stage they were clearly observable as clusters (Fig. 2Biii). The signal was strongest in the cytoplasm but also observable in the nucleus. By E14.5, a distinct KPNA2 perinuclear immunosignal was detected in both germ and somatic cells (Fig. 2Biv). XPO1 appeared to be present in all cells of the E12.5 ovary, with a particularly intense nuclear signal (Fig. 2Bxi). By E14.5 this signal was detected most strongly in the cytoplasm of both germ cells and somatic cells (Fig. 2Bxii). These results confirm that transcripts for key KPNA $\alpha$ and $\beta$ family members are translated during embryonic ovary development and document remarkable changes in protein expression levels and subcellular localisation that correlate with the onset of meiosis. The cellular localisation patterns of KPNA3, KPNB1 and IPO5 proteins are in agreement with previously reported data (Hogarth et al. 2007) and were included for the purpose of consistency.

\section{Dynamic KPNA expression during folliculogenesis}

To determine whether KPNAs are also expressed during postnatal folliculogenesis, we analysed the GSE3351 data series (Pan et al. 2005; Supplementary Table S2) that includes transcripts in oocytes isolated from primordial, 
A

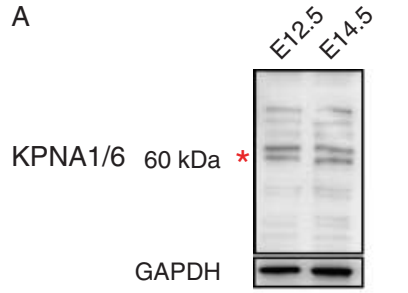

KPNA2

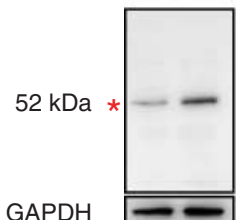

GAPDH

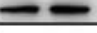

KPNA3

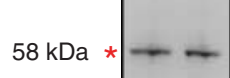

GAPDH
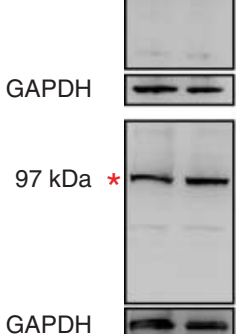

GAPDH

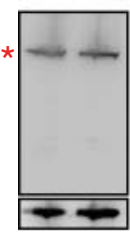

GAPDH

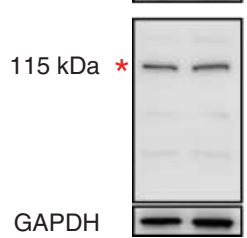

B
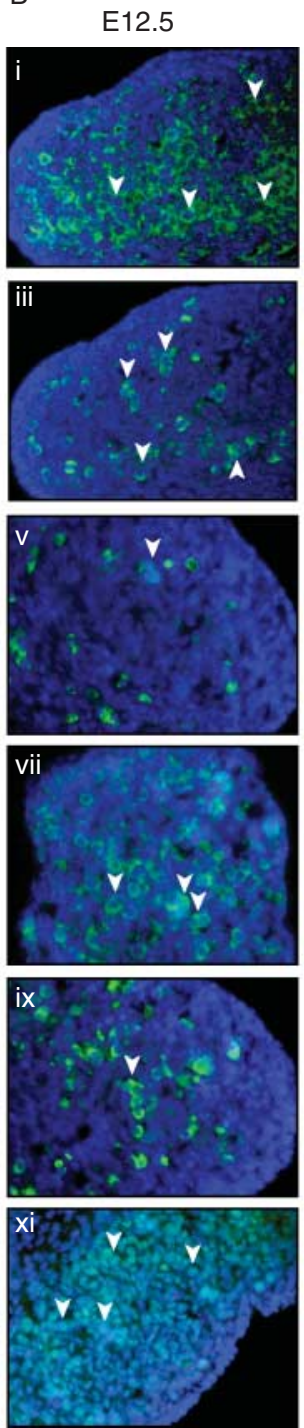
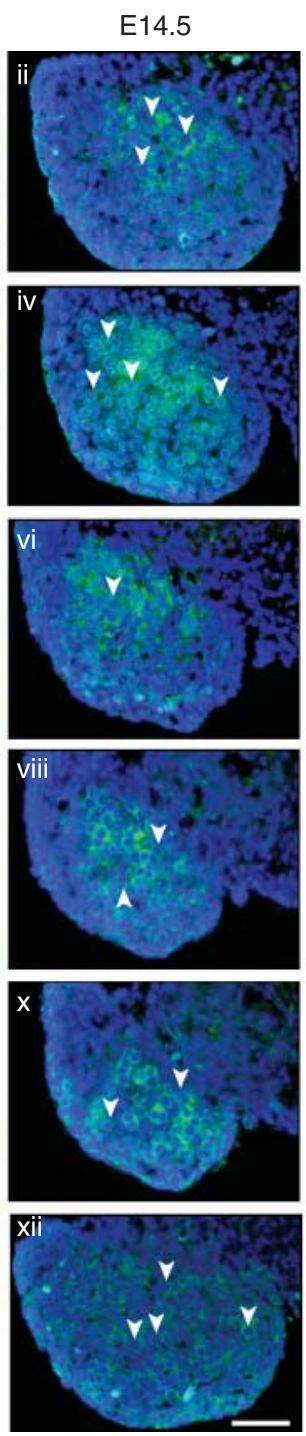

C
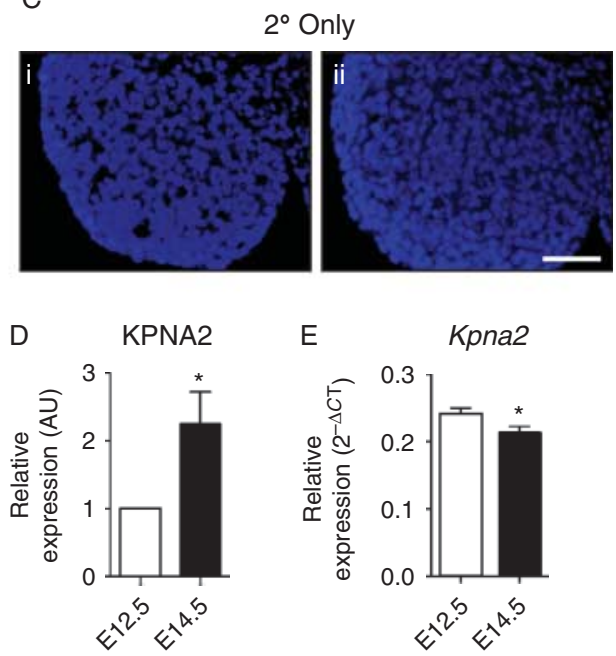

Figure 2 Expression of karyopherin proteins in mouse embryonic ovaries at E12.5 and E14.5. (A) Representative immunoblots for KPNA1/6, KPNA2, KPNA3, KPNB1, IPO5 and XPO1. *, shows protein band. For KPNA1/6 we predict that the two clear bands $~ 60$ kDa represent both KPNA1 and KPNA6 individually expressed at similar levels. Three immunoblots were performed from three different embryonic gonads. (B) Immunolocalisation of KPNA1/6 (i,ii), KPNA2 (iii,iv), KPNA3 (v,vi), KPNB1 (vii,viii), IPO5 (ix, x) and XPO1 (xi, xii) in the embryonic ovary at E12.5 and E14.5 respectively. Nuclei are counterstained with 4',6-diamidino-2-phenylindole (DAPI, blue). Arrows indicate germ cells. (C) Secondary antibody only controls of donkey anti-rabbit Alexa Fluor 594 (i) and donkey anti-goat Alexa Fluor 594 (ii). Scale bars $=100 \mu \mathrm{m}$. (D) Mean relative KPNA2 expression at E14.5 compared to E12.5, normalised to GAPDH and measured in arbitrary units (AU). Error bar shows S.D. from three biological replicates $(P=0.0103$; Student's $t$-test). (E) Relative Kpna2 expression at E12.5 compared to E14.5 via RT-PCR. Error bar shows S.D. from three biological and technical replicates $(P=0.0053$; Student's $t$-test).

primary, secondary, small antral and large antral follicles. Owing to the large differences in expression levels between KPNA $\alpha$ family members, outputs were separated into KPNAs with high (Kpna2, Kpna3 and Kpna7; Supplementary Figure $2 \mathrm{~A}$, see section on supplementary data given at the end of this article) and low expression levels (Kpna1, Kpna4 and Kpna6; Supplementary Figure 2B). Kpna2 displayed high expression across all follicle stages with maximal levels at the primordial and antral stages (Supplementary Figure 2A). Kpna7 increased approximately sevenfold between primordial and primary follicle stages and remained high in secondary and antral follicles (Supplementary Figure 2A). Similarly, Xpo1 increased between primordial and primary follicle stages but subsequently decreased during follicle maturation (Supplementary Figure 2C). In contrast, Kpna1, Kpna2, Kpna4, Kpna6 and Ipo5 decreased after the primordial follicle stage but remained detectable at all other follicle stages during oocyte growth. Only two KPNAs steadily increased during follicle growth: Kpna3 and Kpnb1 (Supplementary Figure $2 \mathrm{~A}$ and $\mathrm{C}$ ). 
A

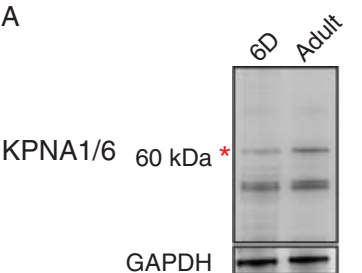

KPNA2

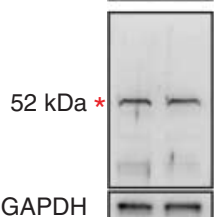

KPNA3

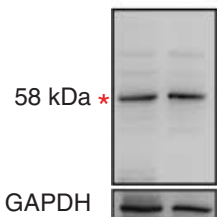

GAPDH

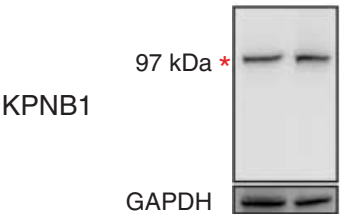

IPO5

XPO1

GAPDH
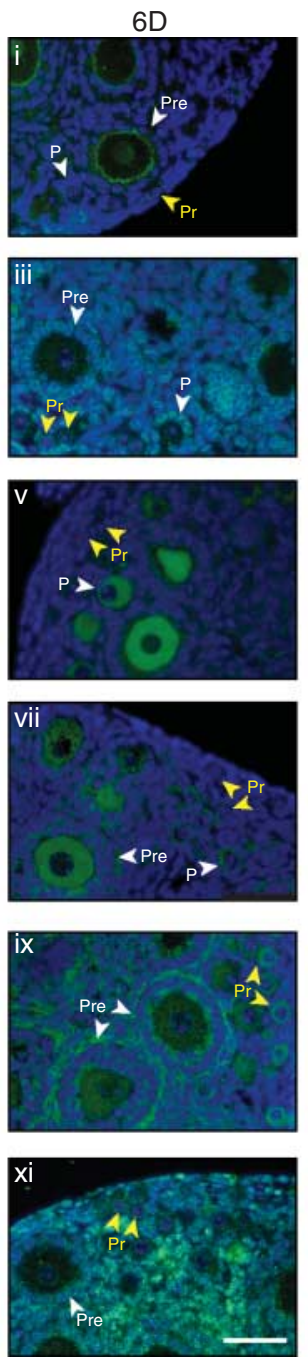

Adult
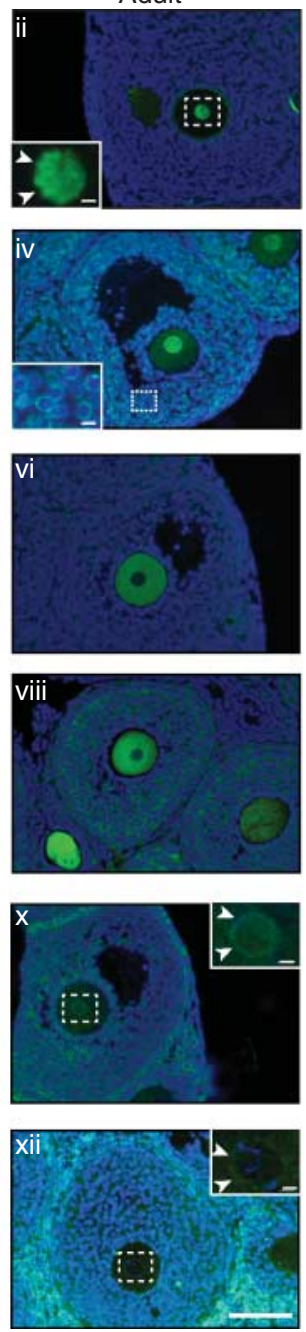

C

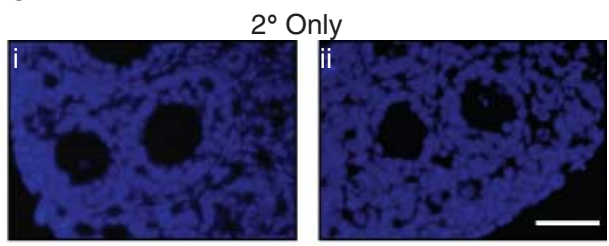

Figure 3 Localisation of karyopherin proteins in the neonatal and adult mouse ovary. (A) Representative immunoblots for KPNA1/6, KPNA2, KPNA3, KPNB1, IPO5 and XPO1. Three immunoblots were performed in biological triplicate. *, shows protein band. (B) Immunolocalisation of KPNA1/6 (i,ii), KPNA2 (iii, iv), KPNA3 (v,vi), KPNB1 (vii,viii), IPO5 (ix, x) and XPO1 (xi,xii) in neonatal and adult mouse ovary respectively. Yellow arrowheads: Pr, primordial follicle; White arrow heads: P, primary follicle; Pre, preantral. Insets show granulosa perinuclear signal (KPNA2); nuclear and perinuclear signal in oocytes (KPNA1/6 and IPO5) and perinuclear signal in oocytes (XPO1). Scale bar: i, iii, v, vii, ix and xi $=50 \mu \mathrm{m}$; ii, iv, vi, viii, $\mathrm{x}$ and $\mathrm{xii}=100 \mu \mathrm{m}$; insets $10 \mu \mathrm{m}$. Nuclei are counterstained with 4',6-diamidino-2-phenylindole (DAPI, blue). (C) Secondary antibody only controls of donkey anti-rabbit Alexa Fluor 594 (i) and donkey anti-goat Alexa Fluor 594 (ii). Scale bar: i and iii $=50 \mu \mathrm{m}$.

We next confirmed the protein expression of KPNA1/6, KPNA2, KPNA3, KPNB1, IPO5 and XPO1 using western blotting in postnatal day $6(6 \mathrm{D})$ and adult ovarian tissue (Fig. 3A). No significant differences in KPNA expression were detected been $6 \mathrm{D}$ and adult ovary. We then performed immunofluorescence analysis to define subcellular localisation. As such, 6D and adult ovarian tissue were used to assess primordial, primary, preantral (two or more layers of granulosa cells) and antral stage follicles. All KPNAs examined were detected at each stage of folliculogenesis: however, we noted distinct differences in subcellular localisation in both the oocytes and granulosa cells (Fig. 3B). Faint cytoplasmic KPNA1/6 and KPNA2 immunostaining was evident in oocytes from the primordial follicle to preantral follicle stage, with strong nuclear enrichment by the antral follicle stage (Fig. 3Bi, ii, iii and iv). Intense KPNA2 perinuclear staining was evident in the granulosa cells of follicles of all stages. In contrast, KPNA3 displayed a strong and exclusively cytoplasmic immunosignal from the primary follicle through to the antral stage, with additional low but detectable cytoplasmic granulosa cell signal present throughout follicle growth (Fig. 3Bv and vi).

The KPNA $\beta$ s, KPNB1 and IPO5, were both primarily cytoplasmic in oocytes and granulosa cells throughout growth; however, IPO5 also became perinuclear and nuclear in antral stage oocytes (Fig. 3Bvii, viii, ix and x). 
Notably, the IPO5 signal was highest in the cytoplasm of primordial stage follicles, which reflects the high IPO5 transcript expression detected at this stage (Supplementary Figure 2C). XPO1 was also predominantly cytoplasmic in oocytes throughout growth but with additional perinuclear staining in all stage follicles with nuclear and cytoplasmic staining of granulosa cells (Fig. 3xi and xii). Therefore, in agreement with the GEO transcript profiles, this immunofluorescence analysis revealed that key members of the KPNAs are present throughout postnatal oocyte development, and differential localisation of each is associated concomitant with the appearance of distinct developmental follicular stages.

\section{KPNA expression in the fully grown GV oocyte and MII egg}

In preparation for fertilisation, the oocyte remains arrested at prophase I in the mature antral (pre-ovulatory) follicle until a luteinizing hormone (LH) surge promotes nuclear envelope breakdown and meiotic progression through to MII. RT-PCR confirmed that all KPNAs examined were expressed in oocytes at these stages with the exception of Kpna4 (Fig. 4). Transcript profiles were then generated using the GEO data set GSE5668 (Su et al. 2007; Supplementary Table S1) that sampled isolated and denuded prophase I arrested GV oocytes and MII eggs (Supplementary Figure 3, see section on supplementary data given at the end of this article).
Transcripts for all KPNAs examined were identified in both GV and MII eggs except for Kpna1 (Supplementary Figure 3). However, we did detect Kpna1 expression using RT-PCR validation on isolated GV and MII eggs (Fig. 4). While Kpna4 transcript was not detected via RT-PCR, GEO transcript levels were the lowest of all of the KPNAs (Supplementary Figure 3).

Using immunoblotting, we were able to detect strong signals for KPNA1/6, KPNA2, KPNA3, KPNB1, IPO5 and XPO1 in GV and MII eggs. Global transcript degradation occurs on transition of the oocyte from GV arrest to MII, yet surprisingly we noted either no significant decrease (Kpna2, Kpna4, Kpna6 and Ipo5) or <20\% loss (Kpna3, Kpna7, Kpnb1 and Xpo1) for these transcripts as was recorded in the GEO data (Supplementary Figure 3). This stability was reflected at the protein level, because immunoblotting showed either a similar signal in MII eggs compared with the GV stage for all KPNAs or a slightly increased signal in the case of KPNA2 and IPO5 (Fig. 5A, D and E).

Immunofluorescence analysis revealed distinct subcellular localisation patterns between the KPNAs at the GV and MII stages (Fig. 5B). In GV oocytes, KPNA2, KPNB1 and IPO5 displayed cytoplasmic and nuclear rim staining (Fig. 5Biii, vii and ix), whereas KPNA1/6 was predominantly cytoplasmic (Fig. 5Bi). For KPNA2, this pattern represented a striking shift from the strong NLS present in preantral and antral stage oocytes (Figs 3Biv and 5Biii). KPNA3 had a particularly prominent signal at the oocyte
Kpna1

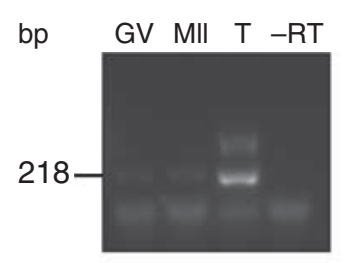

Kpna4

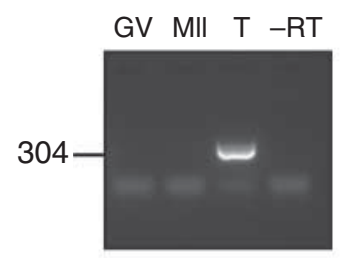

Kpnb1

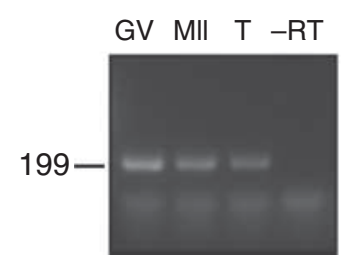

Kpna2

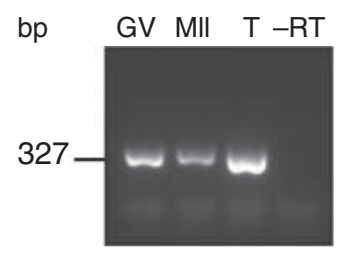

Kpna6

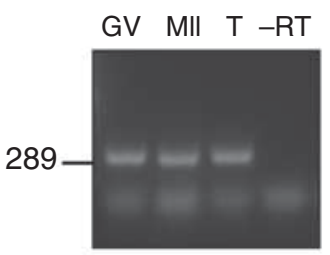

Ipo5

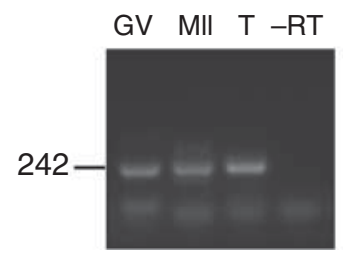

Kpna3

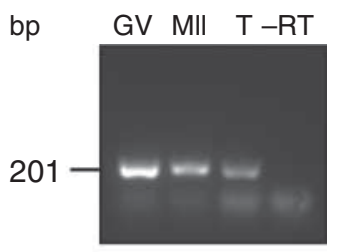

Kpna7

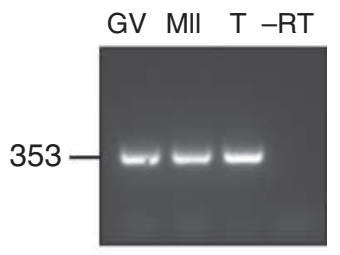

Xpo1

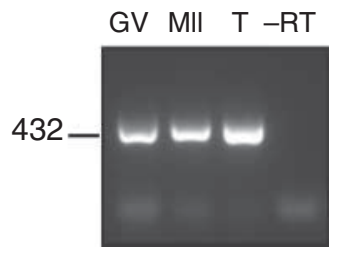

Figure 4 Karyopherin expression in the fully grown GV oocyte and MII egg. Representative RT-PCRs presented for Kpna1, Kpna2, Kpna3, Kpna4, Kpna6, Kpna7, Kpnb1, Ipo5 and Xpo1. RT-PCRs were performed in biological triplicate. T, testis (positive control cDNA); RT, minus RT negative control. 
A

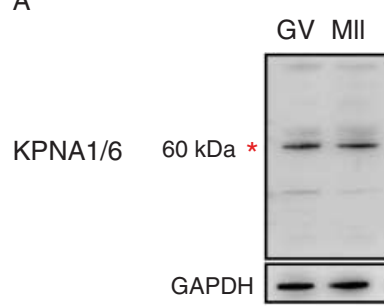

KPNA2

KPNA3

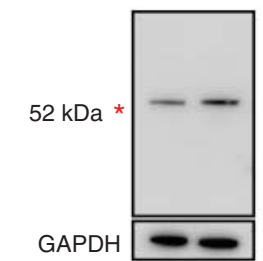

KPNB1

PO5

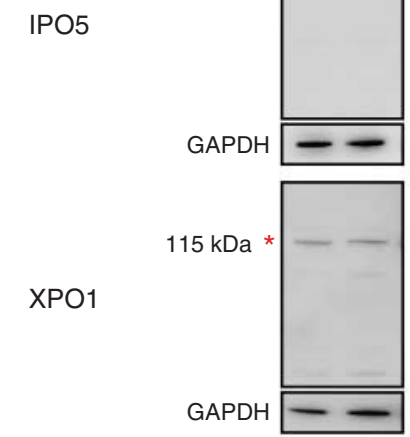

B
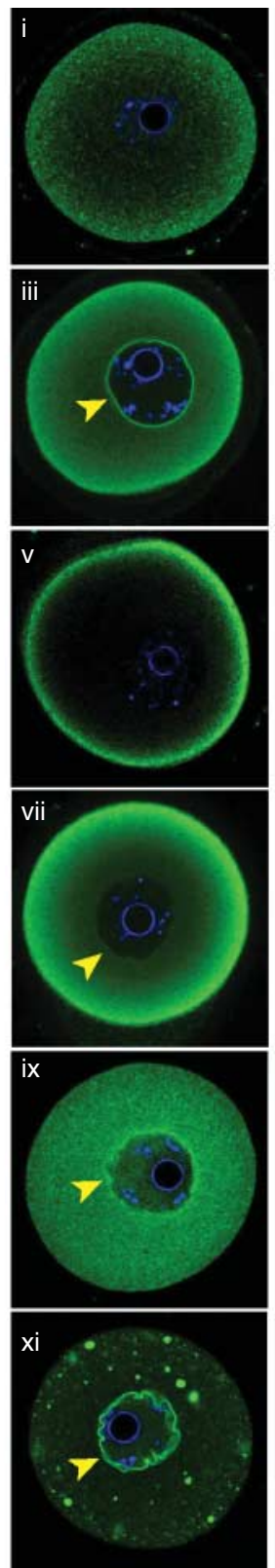

MII
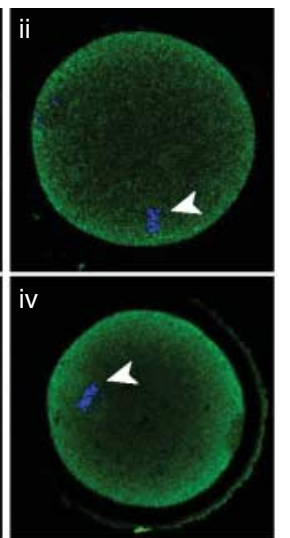

vi
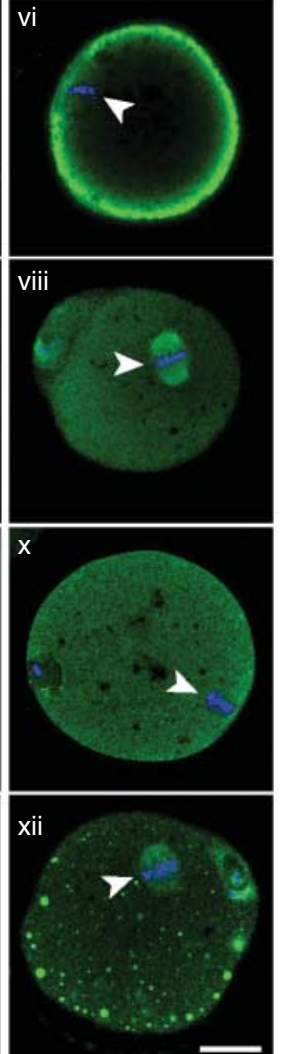

C
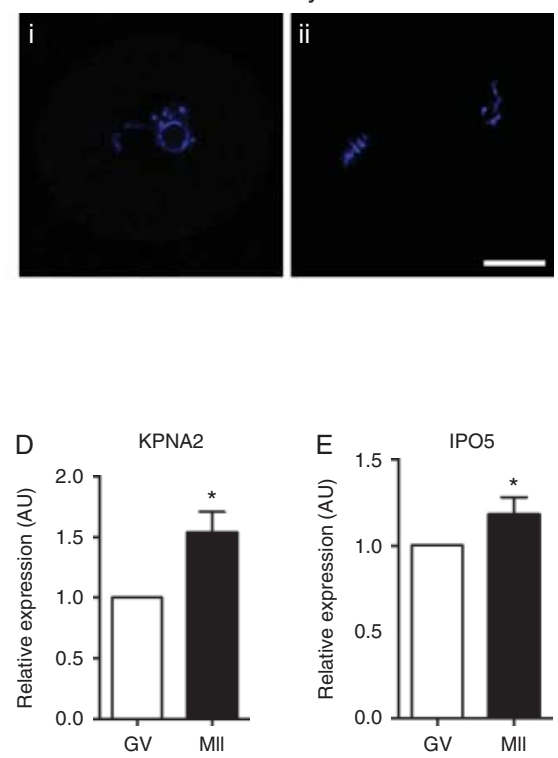

Figure 5 Subcellular localisation of karyopherin (KPNA) proteins in the GV and MII mouse egg. (A) Representative immunoblots for KPNA1/6, KPNA2, KPNA3, KPNB1, IPO5 and XPO1. Based on the transcript expression shown in Supplementary Figure 3, the strongest band present at $\sim 60 \mathrm{kDa}$ for the KPNA1/6 immunoblot may represent KPNA6 rather than KPNA1. KPNA3 blot arrow indicates non-specific bands present in oocytes/eggs. GV, germinal vesicle oocyte; MII, meiosis II ovulated egg. Three immunoblots were performed for each KPNA, 50 oocytes/lane pooled from two mice each. GAPDH was probed as a protein-loading control. *, shows protein band. (B) Representative confocal images showing immunolocalisation of KPNA1/6 (i,ii), KPNA2 (iii,iv), KPNA3 (v,vi), KPNB1 (vii,viii), IPO5 (ix,x) and XPO1 (xi, xii) in GV and MII mouse eggs respectively. Chromatin is counterstained with Hoechst (blue). Yellow arrows indicate nuclear rim of GV oocytes and white arrows show chromatin aligned on MII spindle. (C) Secondary antibody only controls for donkey anti-rabbit Alexa Fluor 633 (i) and donkey anti-goat Alexa Fluor 633 (ii). Scale bar $=20 \mu \mathrm{m}$. (D) Mean relative KPNA2 and (E) IPO5 expression at MII compared to GV, normalised to GAPDH and measured in arbitrary units (AU). Error bar shows S.D. (KPNA2, $P=0.01$ and IPO5, $P=0.03$; Student's $t$-test).

cortex (Fig. 5Bv). Intriguingly, in addition to intense nuclear rim staining, XPO1 was detected in cytoplasmic aggregates (Fig. 5Bxi). At the MII stage (Fig. 5B, all right-hand panels), all of the KPNAs were dispersed throughout cytoplasm except for KPNA3 and XPO1, which retained strong cortex and cytoplasmic aggregate localisation respectively (Fig. 5Bvi and xii). In addition, KPNB1 and XPO1 both localised strongly to the MII spindle (Fig. 5Bviii and xii). 


\section{Discussion}

With their ability to bind distinct sets of cargoes, the KPNAs represent an important class of post-translational regulators that are emerging as mediators of cell development and fate (Poon \& Jans 2005, Yasuhara et al. 2009, Major et al. 2011). Knockout mouse models of a small number of KPNAs implicate them in female germline development (Hu et al. 2010, Moriyama et al. 2011, Rother et al. 2011), and here we have confirmed that KPNAs are widely and differentially expressed during female germ cell development in the mouse, implying they play important roles during oocyte differentiation (Fig. 6).

Transcriptional profiling revealed the expression of six key KPNA $\alpha$ and three KPNA $\beta$ family members in the embryonic germ cells of the mouse ovary. Interestingly, we noted that transcript levels for all KPNAs examined remained relatively stable over this period during which female germ cells transition from mitotic primordial germ cells to meiotic oogonia. This is somewhat in contrast to observations in the embryonic testis where specific KPNAs, such as Kpna2, Kpna3 and Kpna4, display dynamic expression patterns around the time of sex differentiation (Major et al. 2011), although notably these studies investigated whole embryonic testis rather than isolated germ cells and such patterns may reflect changes in the somatic cells also present.
Examination of KPNA proteins in the embryonic ovary, however, revealed a more complex pattern of expression than indicated by their transcript profiles. We found that KPNA2 protein was up-regulated in the female gonad approximately twofold over the period when germ cells start to enter meiosis, yet transcript was slightly decreased. One possible explanation is that translation is up-regulated in the germ cells upon meiotic entry. However, as western blotting was performed on whole gonad tissue rather than isolated germ cells, it may also be possible that the protein increase is a reflection of increased transcript expression in the somatic cells of the female gonad at this time. However, whichever the correct scenario, when taken together with our observations that KPNA2 protein moved from a predominantly cytoplasmic location to become perinuclear, our data supports the theory that this KPNA plays a major role in the events associated with meiotic entry. The equivalent developmental period in the male is the transition from a mitotic spermatogonium to a meiotic spermatocyte, which occurs postnatally, and interestingly, KPNA2 is also up-regulated between these stages in isolated mouse male germ cells at least at the transcript level (Major et al. 2011). A recent measurement of KPNA2 in isolated rat spermatocytes and spermatids demonstrated a significant decrease in the level of this protein as meiotic cells transition to a haploid state (Arjomand et al. 2014). This phenomenon has also been linked to the transition

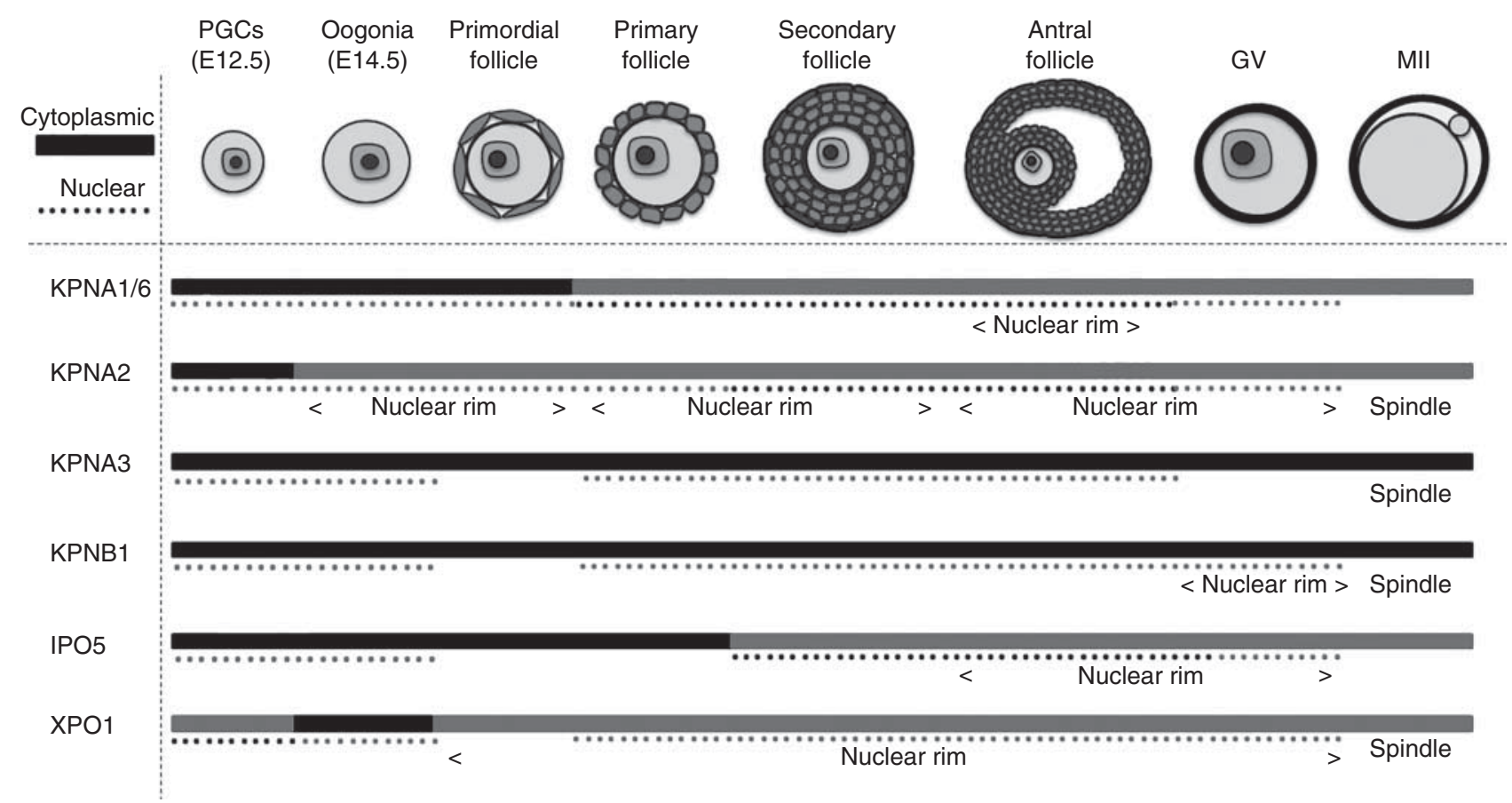

Figure 6 Subcellular distribution of karyopherins throughout murine oogenesis. Diagrammatic representation of the intracellular localisation of KPNA1/6, KPNA2, KPNA3, KPNB1, IPO5 and XPO1 in the developing oocyte throughout folliculogenesis including, primordial germ cells (PGCs) (E12.5), oogonia (E14.5) and primordial, primary, secondary and antral follicle stages as well as in fully grown GV oocyte and MII egg. Solid and dotted lines represent cytoplasmic and nuclear localisation respectively. Relative intracellular localisation intensity within the growing oocyte is also represented using shading: white (no signal) to black (high signal). Nuclear rim and spindle localisations are also notated. 
from undifferentiated state into neural lineage for embryonic stem cells (Yasuhara et al. 2013); hence, identification of KPNA2 binding partners may reveal how meiotic entry and exit is controlled in both sexes.

Although total protein levels did not vary for the other KPNAs examined, their dynamic localisation patterns suggest changing roles at this time. For example, XPO1 became restricted to the cytoplasm of germ and somatic cells by E14.5. A key candidate cargo proposed for XPO1 is the essential meiotic entry factor STRA8, which possesses both NLS and NES sequences and displays dependence on XPO1 for its nuclear-cytoplasmic shuttling (Baltus et al. 2006, Tedesco et al. 2009). When considered together with the re-localisation of other KPNAs, our findings support a model whereby KPNAs regulate meiotic entry through appropriate relocation of important cell cycle regulators. We speculate that changing localisation of KPNAs in fixed cells correlates with either a shift in the function of the KPNA, e.g., from classical nuclear transport to a non-transport role or a shift in the cellular requirement for nucleo-cytoplasmic shuttling of a specific cohort of cargoes. In the case of the developing rodent germ cell, a range of cargos has been identified as being discretely associated with particular KPNAs at distinct developmental stages (Ly-Huynh et al. 2011, Miyamoto et al. 2012, Arjomand et al. 2014, Sutherland et al. 2015). Many of these cargos are not nuclear-localised proteins, and thus the potential for KPNAs to direct binding partners to non-nuclear locations is important to consider.

\section{Postnatal oocyte growth is regulated by KPNAs}

Because the pool of ovarian primordial follicles is finite, the balance between activation and quiescence dictates the reproductive lifespan of a female; remarkably, our knowledge of signalling pathways that govern follicular activation is limited. A small number of oocyteexpressed transcription factors have been identified as regulators of follicle activation, including NOBOX, FOXO3, SOHLH1/2 and LHX8, that may be regulated by nucleo-cytoplasmic transport (reviewed in McLaughlin \& Mclver (2009) and Jagarlamudi \& Rajkovic (2012)). For example, FOXO3A is required to prevent premature follicle activation, and it has been reported to translocate from the nucleus to the cytoplasm following phosphorylation around the time of the primordial to primary follicle transition (Castrillon et al. 2003, Hosaka et al. 2004, John et al. 2008). This highlights the importance of understanding how nuclear access is controlled during oogonial transitions. All KPNAs examined in this study were detected in the primordial follicle, which supports their potential to regulate the crucial mammalian ovarian reserve. The most striking observation was that all KPNAs showed a significant change in expression between the primordial and primary follicle stage, with some down-regulated (Kpna1, Kpna2, Kpna4, Kpna6 and Ipo5) and others up-regulated (Kpnb1, Kpna7 and Xpo1). Although the subcellular localisation of these proteins in these early follicle stages was relatively consistent, these results nonetheless suggest that the changes in KPNA expression are likely to play a role in regulating primordial follicle activation.

The detection of KPNAs at the primary, preantral and antral stages additionally suggests their functional importance for the events associated with oocyte growth, as transcripts and proteins required for oocyte and early embryo development accumulate. Oocyte growth is also associated with a significant remodelling of the oocyte chromatin through DNA methylation via histone acetylation and methylation, culminating with the formation of a transcriptionally quiescent oocyte nucleus at the mature GV stage (Zuccotti et al. 2013). We predict that the nuclear enrichment of KPNA2 and XPO1 in the mature antral oocyte may relate to these epigenetic events. In agreement with this hypothesis, KPNA2 has been shown to bind and transport the chromatin remodelling protein, the protein inhibitor of activated STAT2 (PIAS2), during spermatogenesis, and so it may also be a cargo in the oocyte in which it is also expressed (Ly-Huynh et al. 2011).

Importantly, protein localisation in GV oocytes differed from immature oocytes within ovarian follicles and may reflect the specific cell cycle status of the oocyte at this stage. To maintain meiotic arrest until the appropriate hormonal cues at ovulation, nuclear maturation promoting factor activity (a dimer of CDK1cyclin B1) is suppressed by the nuclear presence of CDK1-inactivating kinase WEE1B, nuclear exclusion of CDK1-activating phosphatase CDC25 and nuclear activity of the E3 ubiquitin ligase complex anaphase promoting complex/Fizzy related 1, which promotes degradation of cyclin B1 (Holt et al. 2010, 2013, Oh et al. 2010). The spatial rearrangement of these cell cycle regulators at the onset of meiotic resumption could be regulated by KPNA-mediated nucleo-cytoplasmic shuttling. Cyclin B1, for example, has been identified as a KPNB1 cargo in somatic cells (Moore et al. 1999, Takizawa et al. 1999, Chook \& Suel 2011). In addition to classical transport roles, the cortex location of KPNA3 and the cytoplasmic vesicle location of XPO1 suggests KPNAs may have other roles in the GV oocyte.

We can also infer that KPNAs are likely to be essential for the MII egg and/or fertilisation and pre-implantation development, because proteins levels appeared to remain stable or slightly increased between the GV to MII stage, despite much of the oocyte transcriptome reportedly being degraded around this time (Paynton \& Bachvarova 1994, Su et al. 2007). In keeping with this concept, we noted localisation of several KPNAs to the MII spindle, which together with the absence of a nuclear envelope at this stage, implies they function in addition to nuclear transport. Roles for KPNAs in spindle assembly and chromosome alignment have been reported for human somatic cells and in Xenopus egg 
extracts. For example, KPNB1 binds and inhibits NLScontaining spindle assembly factors such as NUMA (Xenopus) and TPX2 (Xenopus/human), until RanGTP binding releases them to allow them to promote spindle assembly (Gruss et al. 2001, Nachury et al. 2001, Wiese et al. 2001, Ciciarello et al. 2004). Our results suggest that mammalian meiotic spindle assembly may be also be regulated in a similar manner by the KPNAs.

In addition to oocyte profiling, we were also able to identify widespread expression of KPNAs in the granulosa cell population throughout all follicle stages. Although follicle development may be regarded as being primarily driven by the oocyte, communication with granulosa cells is essential for development of competent oocyte (Eppig 2001, Nilsson \& Skinner 2001). Our data indicates that nucleo-cytoplasmic transport is likely to be essential for multiple aspects of ovarian function.

In conclusion, this study has revealed for the first time that KPNAs are widely expressed at the transcript and protein levels through oocyte development in the mouse. Dynamic changes in transcript and protein expression profiles signify distinct functions for different KPNAs at key stages of oocyte development including meiotic entry, primordial follicle activation and completion of the meiotic divisions (Fig. 6). Future studies will investigate the stage-specific functional roles of specific KPNAs and other nuclear transport factors, as well as identify KPNA-specific cargoes and binding proteins.

\section{Supplementary data}

This is linked to the online version of the paper at http://dx.doi. org/10.1530/REP-14-0585.

\section{Declaration of interest}

The authors declare that there is no conflict of interest that could be perceived as prejudicing the impartiality of the research reported.

\section{Funding}

This work was supported by Australian Research Council (ARC) grants to E A McLaughlin (DP110100418) and J E Holt (DP120100946/DE120101242) and National Health and Medical Research Council grants to K L Loveland (ID1079646). This study was supported in part by the Victorian Government's Operational Infrastructure Support Program.

\section{Acknowledgements}

The authors would like to acknowledge Kate A Redgrove, Jessie M Sutherland and Nicole J Camlin for contributions to the manuscript.

\section{References}

Arjomand A, Baker MA, Li C, Buckle AM, Jans DA, Loveland KL \& Miyamoto Y 2014 The $\alpha$-importome of mammalian germ cell maturation provides novel insights for importin biology. FASEB Journal 28 3480-3493. (doi:10.1096/fj.13-244913)

Baltus AE, Menke DB, Hu YC, Goodheart ML, Carpenter AE, de Rooij DG \& Page DC 2006 In germ cells of mouse embryonic ovaries, the decision to enter meiosis precedes premeiotic DNA replication. Nature Genetics 38 1430-1434. (doi:10.1038/ng1919)

van den Bergen JA, Miles DC, Sinclair AH \& Western PS 2009 Normalizing gene expression levels in mouse fetal germ cells. Biology of Reproduction 81 362-370-418. (doi:10.1095/biolreprod.109.076224)

Castrillon DH, Miao L, Kollipara R, Horner JW \& DePinho RA 2003 Suppression of ovarian follicle activation in mice by the transcription factor Foxo3a. Science 301 215-218. (doi:10.1126/science.1086336)

Chook YM \& Suel KE 2011 Nuclear import by karyopherin- $\beta$ s: recognition and inhibition. Biochimica et Biophysica Acta 1813 1593-1606. (doi:10. 1016/j.bbamcr.2010.10.014)

Ciciarello M, Mangiacasale R, Thibier C, Guarguaglini G, Marchetti E, Di Fiore B \& Lavia P 2004 Importin $\beta$ is transported to spindle poles during mitosis and regulates Ran-dependent spindle assembly factors in mammalian cells. Journal of Cell Science 117 6511-6522. (doi:10.1242/jcs.01569)

Ems-McClung SC, Zheng Y \& Walczak CE 2004 Importin $\alpha / \beta$ and Ran-GTP regulate XCTK2 microtubule binding through a bipartite nuclear localization signal. Molecular Biology of the Cell 15 46-57. (doi:10.1091/ mbc.E03-07-0454)

Eppig JJ 2001 Oocyte control of ovarian follicular development and function in mammals. Reproduction 122 829-838. (doi:10.1530/rep.0. 1220829)

Geles KG, Johnson JJ, Jong S \& Adam SA 2002 A role for Caenorhabditis elegans importin IMA-2 in germ line and embryonic mitosis. Molecular Biology of the Cell 13 3138-3147. (doi:10.1091/mbc.E02-020069)

Gorjanacz M, Adam G, Torok I, Mechler BM, Szlanka T \& Kiss I 2002 Importin- $\alpha 2$ is critically required for the assembly of ring canals during Drosophila oogenesis. Developmental Biology 251 271-282. (doi:10.1006/dbio.2002.0827)

Gruss OJ, Carazo-Salas RE, Schatz CA, Guarguaglini G, Kast J, Wilm M, Le Bot N, Vernos I, Karsenti E \& Mattaj IW 2001 Ran induces spindle assembly by reversing the inhibitory effect of importin $\alpha$ on TPX2 activity. Cell 104 83-93. (doi:10.1016/S0092-8674(01)00193-3)

Harel A, Chan RC, Lachish-Zalait A, Zimmerman E, Elbaum M \& Forbes DJ 2003 Importin $\beta$ negatively regulates nuclear membrane fusion and nuclear pore complex assembly. Molecular Biology of the Cell $\mathbf{1 4}$ 4387-4396. (doi:10.1091/mbc.E03-05-0275)

Hirshfield AN 1991 Development of follicles in the mammalian ovary. International Review of Cytology 124 43-101. (doi:10.1016/S00747696(08)61524-7)

Hogarth CA, Calanni S, Jans DA \& Loveland KL 2006 Importin $\alpha$ mRNAs have distinct expression profiles during spermatogenesis. Developmental Dynamics 235 253-262. (doi:10.1002/dvdy.20569)

Hogarth CA, Jans DA \& Loveland KL 2007 Subcellular distribution of importins correlates with germ cell maturation. Developmental Dynamics 236 2311-2320. (doi:10.1002/dvdy.21238)

Holt JE, Ly-Huynh JD, Efthymiadis A, Hime GR, Loveland KL \& Jans DA 2007 Regulation of nuclear import during differentiation; the IMP $\alpha$ gene family and spermatogenesis. Current Genomics 8 323-334. (doi:10.2174/138920207782446151)

Holt JE, Weaver J \& Jones KT 2010 Spatial regulation of APCCdh1-induced cyclin B1 degradation maintains G2 arrest in mouse oocytes. Development 137 1297-1304. (doi:10.1242/dev.047555)

Holt JE, Lane SI \& Jones KT 2013 The control of meiotic maturation in mammalian oocytes. Current Topics in Developmental Biology 102 207-226. (doi:10.1016/B978-0-12-416024-8.00007-6)

Hosaka T, Biggs lii WH, Tieu D, Boyer AD, Varki NM, Cavenee WK \& Arden KC 2004 Disruption of forkhead transcription factor (FOXO) family members in mice reveals their functional diversification. PNAS 101 2975-2980. (doi:10.1073/pnas.0400093101) 
Hu J, Wang F, Yuan Y, Zhu X, Wang Y, Zhang Y, Kou Z, Wang S \& Gao S 2010 Novel importin- $\alpha$ family member Kpna7 is required for normal fertility and fecundity in the mouse. Journal of Biological Chemistry $\mathbf{2 8 5}$ 33113-33122. (doi:10.1074/jbc.M110.117044)

Itman C, Miyamoto Y, Young J, Jans DA \& Loveland KL 2009 Nucleocytoplasmic transport as a driver of mammalian gametogenesis. Seminars in Cell \& Developmental Biology 20 607-619. (doi:10.1016/ j.semcdb.2009.05.002)

Jagarlamudi K \& Rajkovic A 2012 Oogenesis: transcriptional regulators and mouse models. Molecular and Cellular Endocrinology 356 31-39. (doi:10.1016/j.mce.2011.07.049)

Jameson SA, Natarajan A, Cool J, DeFalco T, Maatouk DM, Mork L, Munger SC \& Capel B 2012 Temporal transcriptional profiling of somatic and germ cells reveals biased lineage priming of sexual fate in the fetal mouse gonad. PLoS Genetics 8 e1002575. (doi:10.1371/journal.pgen.1002575)

John GB, Gallardo TD, Shirley LJ \& Castrillon DH 2008 Foxo3 is a PI3K-dependent molecular switch controlling the initiation of oocyte growth. Developmental Biology 321 197-204. (doi:10.1016/j.ydbio.2008.06.017)

Livak KJ \& Schmittgen TD 2001 Analysis of relative gene expression data using real-time quantitative pcr and the $2^{-\Delta \Delta C T}$ method. Methods 25 401-408. (doi:10.1006/meth.2001.1262)

Loveland KL, Hogarth C, Szczepny A, Prabhu SM \& Jans DA 2006 Expression of nuclear transport importins $\beta 1$ and $\beta 3$ is regulated during rodent spermatogenesis. Biology of Reproduction 74 67-74. (doi:10.1095/biolreprod.105.042341)

Ly-Huynh JD, Lieu KG, Major AT, Whiley PA, Holt JE, Loveland KL \& Jans DA 2011 Importin $\alpha 2$-interacting proteins with nuclear roles during mammalian spermatogenesis. Biology of Reproduction 85 1191-1202. (doi:10.1095/biolreprod.111.091686)

Macara IG 2001 Transport into and out of the nucleus. Microbiology and Molecular Biology Reviews 65 570-594 (table of contents). (doi:10. 1128/MMBR.65.4.570-594.2001)

Major AT, Whiley PAF \& Loveland KL 2011 Expression of nucleocytoplasmic transport machinery: clues to regulation of spermatogenic development. Biochimica et Biophysica Acta 1813 1668-1688. (doi:10.1016/j.bbamcr.2011.03.008)

Marfori M, Mynott A, Ellis JJ, Mehdi AM, Saunders NF, Curmi PM, Forwood JK, Boden M \& Kobe B 2011 Molecular basis for specificity of nuclear import and prediction of nuclear localization. Biochimica et Biophysica Acta 1813 1562-1577. (doi:10.1016/j.bbamcr.2010.10.013)

McLaughlin EA \& Mclver SC 2009 Awakening the oocyte: controlling primordial follicle development. Reproduction 137 1-11. (doi:10.1530/ REP-08-0118)

Miyamoto Y, Boag PR, Hime GR \& Loveland KL 2012 Regulated nucleocytoplasmic transport during gametogenesis. Biochimica et Biophysica Acta 1819 616-630. (doi:10.1016/j.bbagrm.2012.01.015)

Moore JD, Yang J, Truant R \& Kornbluth S 1999 Nuclear import of $\mathrm{Cdk} /$ cyclin complexes: identification of distinct mechanisms for import of Cdk2/cyclin E and Cdc2/cyclin B1. Journal of Cell Biology 144 213-224. (doi:10.1083/jcb.144.2.213)

Moriyama T, Nagai M, Oka M, Ikawa M, Okabe M \& Yoneda Y 2011 Targeted disruption of one of the importin $\alpha$ family members leads to female functional incompetence in delivery. FEBS Journal $\mathbf{2 7 8}$ 1561-1572. (doi:10.1111/j.1742-4658.2011.08079.x)

Nachury MV, Maresca TJ, Salmon WC, Waterman-Storer CM, Heald R \& Weis K 2001 Importin $\beta$ is a mitotic target of the small GTPase Ran in spindle assembly. Cell 104 95-106. (doi:10.1016/S0092-8674(01)00194-5)

Nilsson E \& Skinner MK 2001 Cellular interactions that control primordial follicle development and folliculogenesis. Journal of the Society for Gynecologic Investigation 8 S17-S20. (doi:10.1016/S1071-5576(00) 00099-X)

Oh JS, Han SJ \& Conti M 2010 Wee1B, Myt1, and Cdc25 function in distinct compartments of the mouse oocyte to control meiotic resumption. Journal of Cell Biology 188 199-207. (doi:10.1083/jcb.200907161)

Pan H, O'Brien MJ, Wigglesworth K, Eppig JJ \& Schultz RM 2005 Transcript profiling during mouse oocyte development and the effect of gonadotropin priming and development in vitro. Developmental Biology 286 493-506. (doi:10.1016/j.ydbio.2005.08.023)

Paynton BV \& Bachvarova R 1994 Polyadenylation and deadenylation of maternal mRNAs during oocyte growth and maturation in the mouse. Molecular Reproduction and Development 37 172-180. (doi:10.1002/ mrd.1080370208)
Poon IK \& Jans DA 2005 Regulation of nuclear transport: central role in development and transformation? Traffic 6 173-186. (doi:10.1111/ j.1600-0854.2005.00268.x)

Rother F, Shmidt T, Popova E, Krivokharchenko A, Hugel S, Vilianovich L, Ridders M, Tenner K, Alenina N, Kohler M et al. 2011 Importin $\alpha 7$ is essential for zygotic genome activation and early mouse development. PLOS ONE 6 e18310. (doi:10.1371/journal.pone.0018310)

Schatz CA, Santarella R, Hoenger A, Karsenti E, Mattaj IW, Gruss OJ \& Carazo-Salas RE 2003 Importin $\alpha$-regulated nucleation of microtubules by TPX2. EMBO Journal 22 2060-2070. (doi:10.1093/emboj/cdg195)

Stewart M 2007 Molecular mechanism of the nuclear protein import cycle. Nature Reviews. Molecular Cell Biology 8 195-208. (doi:10.1038/ nrm2114)

Su YQ, Sugiura K, Woo Y, Wigglesworth K, Kamdar S, Affourtit J \& Eppig JJ 2007 Selective degradation of transcripts during meiotic maturation of mouse oocytes. Developmental Biology 302 104-117. (doi:10.1016/j. ydbio.2006.09.008)

Sutherland JM, Sobinoff AP, Fraser BA, Redgrove KA, Davidson TL, Siddall NA, Koopman P, Hime GR \& McLaughlin EA 2015 RNA binding protein Musashi-1 directly targets Msi2 and Erh during early testis germ cell development and interacts with IPO5 upon translocation to the nucleus. FASEB Journal 29 2759-2768. (doi:10.1096/fj.14-265868)

Takizawa CG, Weis K \& Morgan DO 1999 Ran-independent nuclear import of cyclin B1-Cdc2 by importin $\beta$. PNAS 96 7938-7943. (doi:10.1073/ pnas.96.14.7938)

Tedesco M, La Sala G, Barbagallo F, De Felici M \& Farini D 2009 STRA8 shuttles between nucleus and cytoplasm and displays transcriptional activity. Journal of Biological Chemistry 284 35781-35793. (doi:10.1074/jbc.M109.056481)

Tirian L, Timinszky G \& Szabad J 2003 P446L-importin- $\beta$ inhibits nuclear envelope assembly by sequestering nuclear envelope assembly factors to the microtubules. European Journal of Cell Biology 82 351-359. (doi:10. 1078/0171-9335-00324)

Whiley PA, Miyamoto Y, McLachlan RI, Jans DA \& Loveland KL 2012 Changing subcellular localization of nuclear transport factors during human spermatogenesis. International Journal of Andrology 35 158-169. (doi:10.1111/j.1365-2605.2011.01202.x)

Wiese C, Wilde A, Moore MS, Adam SA, Merdes A \& Zheng Y 2001 Role of importin- $\beta$ in coupling Ran to downstream targets in microtubule assembly. Science 291 653-656. (doi:10.1126/science.1057661)

Yasuda Y, Miyamoto Y, Yamashiro T, Asally M, Masui A, Wong C, Loveland KL \& Yoneda Y 2012 Nuclear retention of importin $\alpha$ coordinates cell fate through changes in gene expression. EMBO Journal 31 83-94. (doi:10.1038/emboj.2011.360)

Yasuhara N, Oka M \& Yoneda Y 2009 The role of the nuclear transport system in cell differentiation. Seminars in Cell \& Developmental Biology 20 590-599. (doi:10.1016/j.semcdb.2009.05.003)

Yasuhara N, Yamagishi R, Arai Y, Mehmood R, Kimoto C, Fujita T, Touma K, Kaneko A, Kamikawa Y, Moriyama T et al. 2013 Importin $\alpha$ subtypes determine differential transcription factor localization in embryonic stem cells maintenance. Developmental Cell 26 123-135. (doi:10.1016/ j.devcel.2013.06.022)

Young JC, Ly-Huynh JD, Lescesen H, Miyamoto Y, Browne C, Yoneda Y, Koopman P, Loveland KL \& Jans DA 2013 The nuclear import factor importin $\alpha 4$ can protect against oxidative stress. Biochimica et Biophysica Acta 1833 2348-2356. (doi:10.1016/j.bbamcr.2013.06.007)

Zhang C, Hutchins JR, Muhlhausser P, Kutay U \& Clarke PR 2002 Role of importin- $\beta$ in the control of nuclear envelope assembly by Ran. Current Biology 12 498-502. (doi:10.1016/S0960-9822(02)00714-5)

Zuccotti M, Merico V, Redi CA \& Garagna S 2013 An epigenomic biography of the mammalian oocyte. In Oogenesis, end 1, pp 141-150. Eds G Coticchio, DF Albertini \& L De Santis. London: Springer. (doi:10. 1007/978-0-85729-826-3_10)

Received 6 November 2014

First decision 19 December 2014

Revised manuscript received 20 August 2015

Accepted 23 September 2015 